\title{
Multifractal Analysis of Fetal Heart Rate Variability in Fetuses with and without Severe Acidosis during Labor
}

\author{
Muriel Doret, M.D., Ph.D., 1,2 Hannes Helgason, Ph.D.., 3 Patrice Abry, Ph.D., 3 \\ Paulo Goncalves, Ph.D., ${ }^{4}$ Claude Gharib, M.D., Ph.D. ${ }^{2}$ \\ and Pascal Gaucherand, M.D. ${ }^{1,2}$
}

\begin{abstract}
We performed multifractal analysis of fetal heart rate (FHR) variability in fetuses with and without acidosis during labor. Multifractal analysis was performed on fetal electrocardiograms in 10-minute sliding windows within the last 2 hours before delivery in 45 term fetuses divided in three groups according to umbilical arterial $\mathrm{pH}$ and FHR pattern: group $\mathrm{A}$ had $\mathrm{pH} \geq 7.30$ and normal FHR, group $\mathrm{B}$ had $\mathrm{pH} \geq 7.30$ and intermediate or abnormal FHR, and group $\mathrm{C}$ had acidosis $(\mathrm{pH} \leq 7.05)$ and intermediate or abnormal FHR. Six multifractal parameters were compared using Wilcoxon rank sum test. Multifractal parameters were significantly different between the three groups in the last 10 minutes before delivery $(p<0.05)$. Two parameters $\left(b_{\min }\right.$, zeta $\left.(2)\right)$ exhibited a significant difference 70 minutes before delivery, and one parameter $\left(C_{2}\right)$ was different 10 minutes before birth $(p<0.05)$. Multifractal parameters were significantly different in acidotic and nonacidotic fetuses, independently from FHR pattern.
\end{abstract}

KEYWORDS: Acidosis, fetal heart rate, labor, multifractal analysis, variability

F etal surveillance during labor is essential to reduce neonatal mortality and morbidity due to per partum asphyxia. Continuous fetal heart rate (FHR) monitoring is a useful screening test with high sensitivity to detect fetal asphyxia with metabolic acidosis and cerebral palsy. Conversely, its low specificity justifies efforts to develop additional tests to help identifying false-positive tests of FHR visual analysis. ${ }^{1,2}$ Complementary methods, such as fetal scalp $\mathrm{pH}$ and lactates, fetal electrocardiogram (ECG) ST segment analysis, and T/QRS ratio $\left(\mathrm{STAN}^{\circledR}\right.$; Neoventa Medical, Moelndal,
Sweden), have been shown, at the best, to modestly reduce fetal metabolic acidosis and rate of operative deliveries. $^{3-6}$

As FHR with reduced or absent variability is one of the most significant parameters to predict the development of fetal acidosis, 2,7 some researches focused on FHR variability analysis. Objective measurement of heart rate variability can be performed with different methods like computerized analysis based on statistical description (i.e., the well-known short- and long-term variabilities), spectral analysis, entropy, and fractal approaches. ${ }^{8-15} \mathrm{Few}$
${ }^{1}$ Hospices Civils de Lyon, Hôpital Femme-Mère-Enfant, service de gynécologie-obstétrique, Bron; ${ }^{2}$ Université de Lyon, Faculté de Médecine Lyon Est; ${ }^{3}$ Ecole normale neperieure de Lyon, Laboratoire de Physique; ${ }^{4}$ Ecole normale neperieure de Lyon, LIP, INRIA RESO, Lyon, France.

Address for correspondence and reprint requests: Muriel Doret, M.D., Ph.D., Hospices Civils de Lyon, Hôpital Femme-Mère-Enfant, service de gynécologie-obstétrique, 59, boulevard Pinel, Bron cedex
F-69677, France (e-mail: muriel.doret@chu-lyon.fr).

Am J Perinatol 2011;28:259-266. Copyright (C) 2011 by Thieme Medical Publishers, Inc., 333 Seventh Avenue, New York, NY 10001, USA. Tel: +1(212) 584-4662.

Received: July 20, 2010. Accepted after revision: September 20, 2010. Published online: November 18, 2010.

DOI: http://dx.doi.org/10.1055/s-0030-1268713.

ISSN 0735-1631. 
studies, based on these statistical tools, focused on FHR variability analysis during labor to detect hypoxia or acidosis, with variable results. ${ }^{16-21}$

Multifractal analysis is a recent technique that aims at providing detailed and elaborated analysis of data variability, particularly adapted for characterization of nonstationary and complex signals like FHR. ${ }^{22,23}$ Although variability is clinically analyzed by considering the largest difference in data (referred to as "oscillation") observed within a sliding time window of a priori chosen size (usually 1 minute), multifractal analysis focuses on characterizing the evolution of the variability measured in a collection of windows of different growing sizes, within a relevant range that is a posteriori and adaptively determined by the method itself. ${ }^{14,15,22,23}$ In experimental studies, multifractal properties have been shown to be closely related to the cardiovascular control mechanism, and it has been demonstrated that multifractal properties could be used to quantify the autonomic nervous system activity. ${ }^{12,22,23}$ Autonomic nervous system activity physiologically varies according to baroreflex and chemoreflex stimulation and central nervous system response, and is largely responsible for heart rate variability. ${ }^{24}$ Hence, multifractal analysis, in addition to regular FHR monitoring, may be a promising new approach to objectively evaluate FHR variability. Therefore, we conducted a pilot study to explore and compare multifractal parameters of heart rate variability in a population of fetuses with and without acidosis during labor, presenting with different FHR patterns.

\section{METHODS}

\section{Patient Selection}

Cardiotocographic recordings were collected in the Department of Obstetrics at the public academic hospital Femme-Mère-Enfant (Bron, France) between 2000 and 2007. FHR monitorings were recorded during labor with either a STAN S21 or a STAN S31 monitor and collected in a large database. The first 45 consecutive patients meeting the inclusion criteria were included, until each group was composed of 15 patients (see below). Inclusion criteria were low-risk pregnancy, gestational age between 37 and 42 weeks, recording lasting more than 30 minutes that was not stopped more than 30 minutes before delivery and having less than 10\% missing data, with umbilical artery $\mathrm{pH}$ and neonatal outcome documented. Forty-five patients were selected by the first author (M.D.) according to umbilical cord $\mathrm{pH}$ and FHR pattern (based on the "Three-Tier Fetal Heart Rate Interpretation System" established by the National Institute of Child Health and Human Development [NICHD]) and grouped as follows ${ }^{25}$ :
Group A (control): arterial $\mathrm{pH} \geq 7.30$ and normal FHR (category I; $n=15$ );

Group B: arterial $\mathrm{pH} \geq 7.30$ and intermediate or abnormal FHR (categories II and III; $n=15$ );

Group C: acidosis defined as arterial $\mathrm{pH} \leq 7.05$ and intermediate or abnormal FHR (category II and III; $n=15$ ).

All women had epidural analgesia at the time of FHR recording. Labor and delivery management were completed according to the STAN clinical guidelines.

\section{Data Acquisition and Signal Preprocessing}

Fetal ECGs were collected using a scalp electrode and recorded with a STAN S21 or S31 monitor at 12-bit resolution and $500-\mathrm{Hz}$ sampling rate. STAN generates an electronic file for each recording, and the R-R intervals were extracted from the stored STAN files by Neoventa Medical. As commonly done in heart rate analysis, the $\mathrm{R}-\mathrm{R}$ interval data sets were transformed to a continuous signal by linear interpolation and the event series were resampled at the rate of $8 \mathrm{~Hz}$.

\section{Multifractal Analysis}

To characterize FHR variability, multifractal analysis was applied to $\mathrm{R}-\mathrm{R}$ interval time series over 10-minute time windows because this would correspond to the shortest time interval from decision to delivery that can be reasonably achieved in clinical practice. It was first applied to the last 10 minutes before delivery and then applied independently to 10-minute-long sliding windows over the last 120 minutes.

Six multifractal parameters, named $C_{1}, C_{2}, h_{\mathrm{Max}}$, $h_{\min }, \operatorname{zeta}(2)$ and zeta(-2), were extracted to form a concise descriptor set of the multifractal spectrum (Figs. 1 and 2). ${ }^{14,15}$ Roughly, the parameter $C_{1}$ can be viewed as the most common FHR variability within the

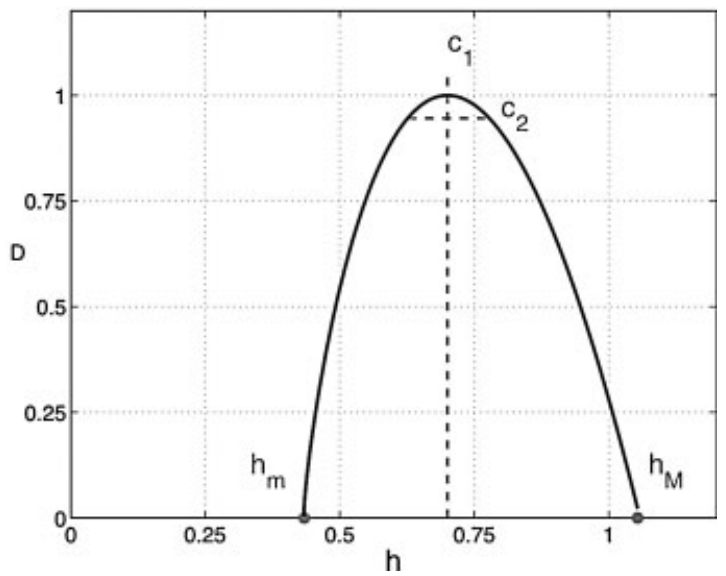

Figure 1 Schematic representation of a typical multifractal spectrum. The parameters $C_{1}, C_{2}, h_{\min }, h_{\mathrm{Max}}$ are represented on the multifractal spectrum. 
A
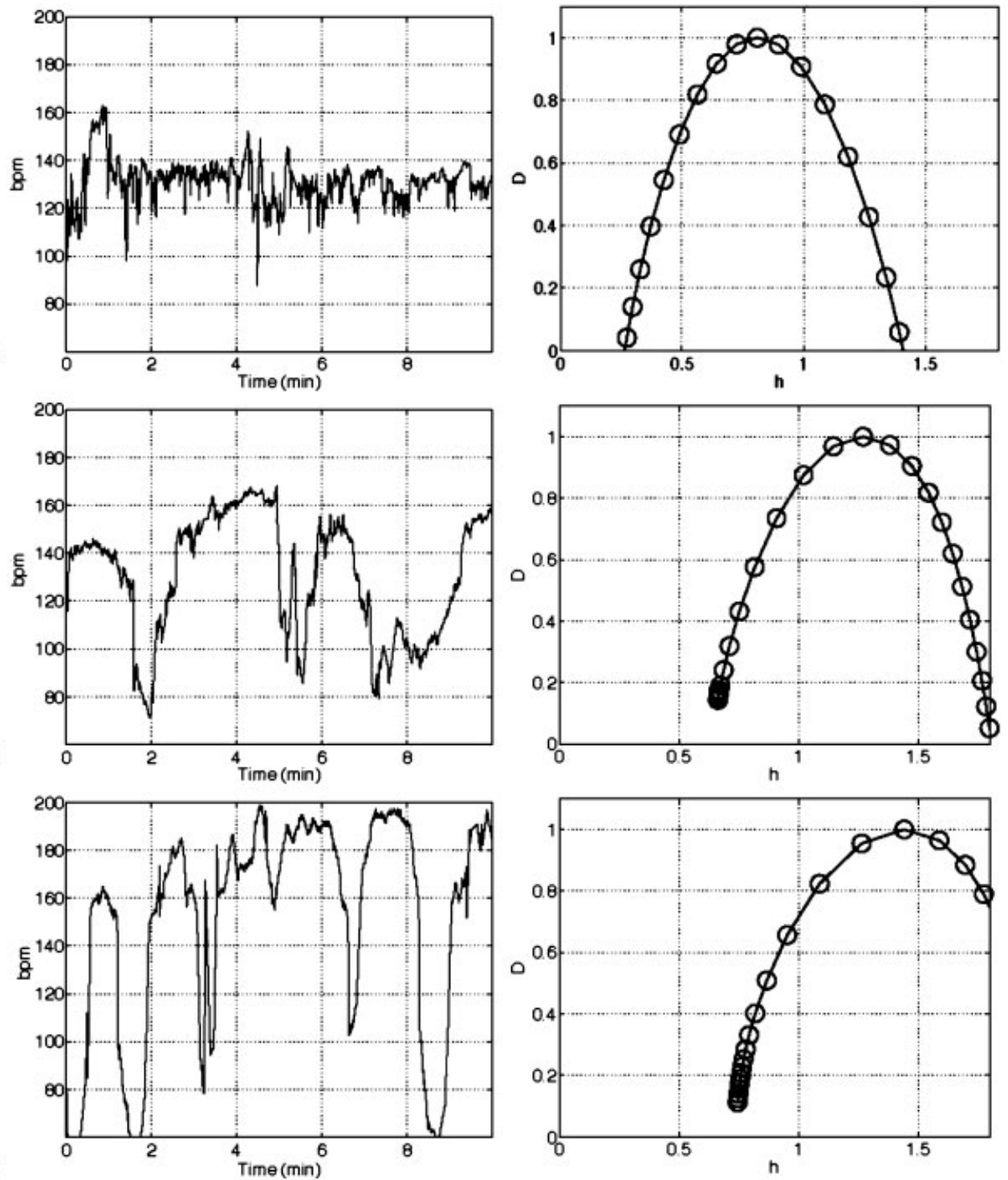

Figure 2 Typical examples of multifractal spectrum. From top to bottom, examples of a multifractal spectrum of fetuses from groups $A, B$, and $C$ computed over the last 10-minute window. bpm, beats per minute.

analyzed window. This parameter is very close to the global variability assessed with visual analysis. An increase in $C_{1}$ indicates a decrease in variability. The parameter $C_{2}$ indicates how the variability departs from the $C_{1}$ value. A small $C_{2}$ denotes a variability that changes little over time. Conversely, a high $C_{2}$ indicates that portions of the signal can differ greatly in variability, compared with the typical $C_{1}$ value. The parameters $h_{\min }$ and $h_{\text {Max }}$ correspond respectively to the largest and smallest variabilities that can be observed in the data. To practically obtain $C_{1}$ and $C_{2}$, it is necessary to compute a set of intermediate parameters zeta $(q)$ (cf. Appendix for details). From these intermediate parameters, zeta(2) was retained as it can be theoretically related to Fourier analysis and spectrum estimation. ${ }^{26}$ The larger zeta(2) is, the smaller the variability in the data is. Zeta(-2) was also included as multifractal analysis theoretically requires that both positive and negative $q$ are used. To summarize, the larger the parameters are, the lower the variability is.

In the present study, multifractal analysis was based on wavelet Leader, a recent method introduced in an article by Wendt et al. ${ }^{27}$ For more information, the reader is referred to the Appendix and to the reviews from Riedi and Jaffard. ${ }^{14,15}$

\section{Statistical Analysis}

Multifractal parameters are expressed as median and absolute median deviation computed over each group. The six multifractal parameters are statistically analyzed individually using the Wilcoxon rank sum tests and jointly using the Bonferroni-Holm standard procedure. Statistical significance for $p$ values was set to $p<0.05$. 
Table 1 FHR Description in the Groups with Intermediate or Abnormal FHR (Categories II and III) and Normal Umbilical Arterial pH (group B) or Acidosis (Group C)

\begin{tabular}{lll}
\hline FHR description & Group B (n=15) & Group C (n= 15) \\
\hline Category II & 3 & 3 \\
Moderate baseline variability + recurrent variable decelerations & 2 & 1 \\
Moderate baseline variability + recurrent late decelerations & 1 & 0 \\
Moderate baseline variability + prolonged decelerations & 5 & 0 \\
Minimal baseline variability & 1 & 5 \\
Minimal baseline variability + recurrent variable decelerations & 1 & 1 \\
Minimal baseline variability + recurrent late decelerations & 0 & 1 \\
Minimal baseline variability + bradycardia & 1 & 1 \\
Minimal baseline variability + tachycardia & & 0 \\
Category III & 1 & 1 \\
Absent variability without any deceleration & 0 & 2 \\
Absent baseline variability accompanied by recurrent variable decelerations & 0 &
\end{tabular}

FHR, fetal heart rate.

\section{RESULTS}

\section{Population Characteristics}

Group A (control group) included fetuses with umbilical arterial $\mathrm{pH} \geq 7.30$ and normal FHR pattern (category I). Mean umbilical arterial $\mathrm{pH}$ was $7.34 \pm 0.03$ (range: 7.30 to 7.40 ). All FHRs exhibited a baseline frequency between 110 and 160 beats per minute (bpm), variability between 6 and $25 \mathrm{bpm}$, and accelerations. No deceleration was noticed in nine cases. Episodic or recurrent early decelerations were present in six cases. Group B included fetuses with umbilical arterial $\mathrm{pH} \geq 7.30$ and intermediate or abnormal FHR (categories II and III) according to the NICHD classification. Mean umbilical arterial $\mathrm{pH}$ was $7.32 \pm 0.02$ (range: 7.30 to 7.40 ). FHR classification is displayed in Table 1. Group $\mathrm{C}$ included fetuses with umbilical arterial $\mathrm{pH} \leq 7.05$ and intermediate or abnormal FHR (Table 1). Mean umbilical arterial $\mathrm{pH}$ was $7.00 \pm 0.03$ (range: 6.95 to 7.05 ).

\section{Multifractal Properties}

For practical use, we checked that the multifractal parameters are statistically consistent when the analyzing wavelet (Daubechies wavelets), the sampling frequency, the durations, and positions of the sliding windows vary. ${ }^{28}$ This provided us with solid evidences in favor of the meaningfulness of the multifractal parameters measured and discussed here. One of the most important outcomes of this study consists of the determination of the window sizes relevant for multifractal analysis from 4 to 64 seconds.

\section{Multifractal Analysis on the Last 10 Minutes before Delivery}

A representative multifractal spectrum for each group is shown in Fig. 2. Median values for multifractal parameters for each group are presented in Table 2. Using the Wilcoxon rank sum test, all six parameters were found to be significantly different in groups $\mathrm{A}$ and $\mathrm{C}$ (all $p \leq 0.006)$ and when comparing groups $\mathrm{B}$ and $\mathrm{C}$ $(p<0.05)$. Parameters systematically took higher values in the group of acidotic fetuses (group C). The standard Bonferroni-Holm procedure showed $p$ as low as 0.02 for group $\mathrm{C}$ versus both group $\mathrm{A}$ and group $\mathrm{B}$. Comparing group $\mathrm{B}$ with group $\mathrm{A}, C_{1}$ was the only parameter to be significantly different $(p<0.05) . C_{2}, h_{\min }, h_{\mathrm{Max}}, \mathrm{zeta}(2)$, and zeta( $(-2)$ were not statistically different $(p>0.05)$.

\section{Multifractal Analysis on the Last 2 Hours before Delivery Using 10-Minute Sliding Windows}

For each patient in the three groups, the six multifractal parameters were computed within consecutive 10-minute-long sliding windows during the last 2 hours before

Table 2 Multifractal Parameters for the Three Groups of Fetuses*

\begin{tabular}{lllllll}
\hline & $\boldsymbol{C}_{\mathbf{1}}$ & $\boldsymbol{C}_{\mathbf{2}}$ & $\boldsymbol{h}_{\min }$ & $\boldsymbol{h}_{\text {Max }}$ & Zeta(2) & Zeta(-2) \\
\hline Group A & $0.90 \pm 0.14$ & $0.12 \pm 0.99$ & $0.39 \pm 0.15$ & $1.27 \pm 2.7$ & $1.46 \pm 0.27$ & $2.03 \pm 5.08$ \\
Group B & $1.12 \pm 0.13$ & $0.16 \pm 1.5$ & $0.45 \pm 0.13$ & $1.44 \pm 1.87$ & $1.76 \pm 0.25$ & $2.4 \pm 3.66$ \\
Group C & $1.4 \pm 0.29$ & $0.3 \pm 4.5$ & $0.7 \pm 0.13$ & $7.2 \pm 3.9$ & $2.17 \pm 0.30$ & $13.17 \pm 7.73$
\end{tabular}

*Group A included fetuses with normal FHR and umbilical arterial $\mathrm{pH} \geq 7.30$; group B included fetuses with intermediate or abnormal FHR and umbilical arterial $\mathrm{pH} \geq 7.30$; group $\mathrm{C}$ included fetuses with intermediate or abnormal $\mathrm{FHR}$ and umbilical arterial $\mathrm{pH} \leq 7.05$. FHR, fetal heart rate. 

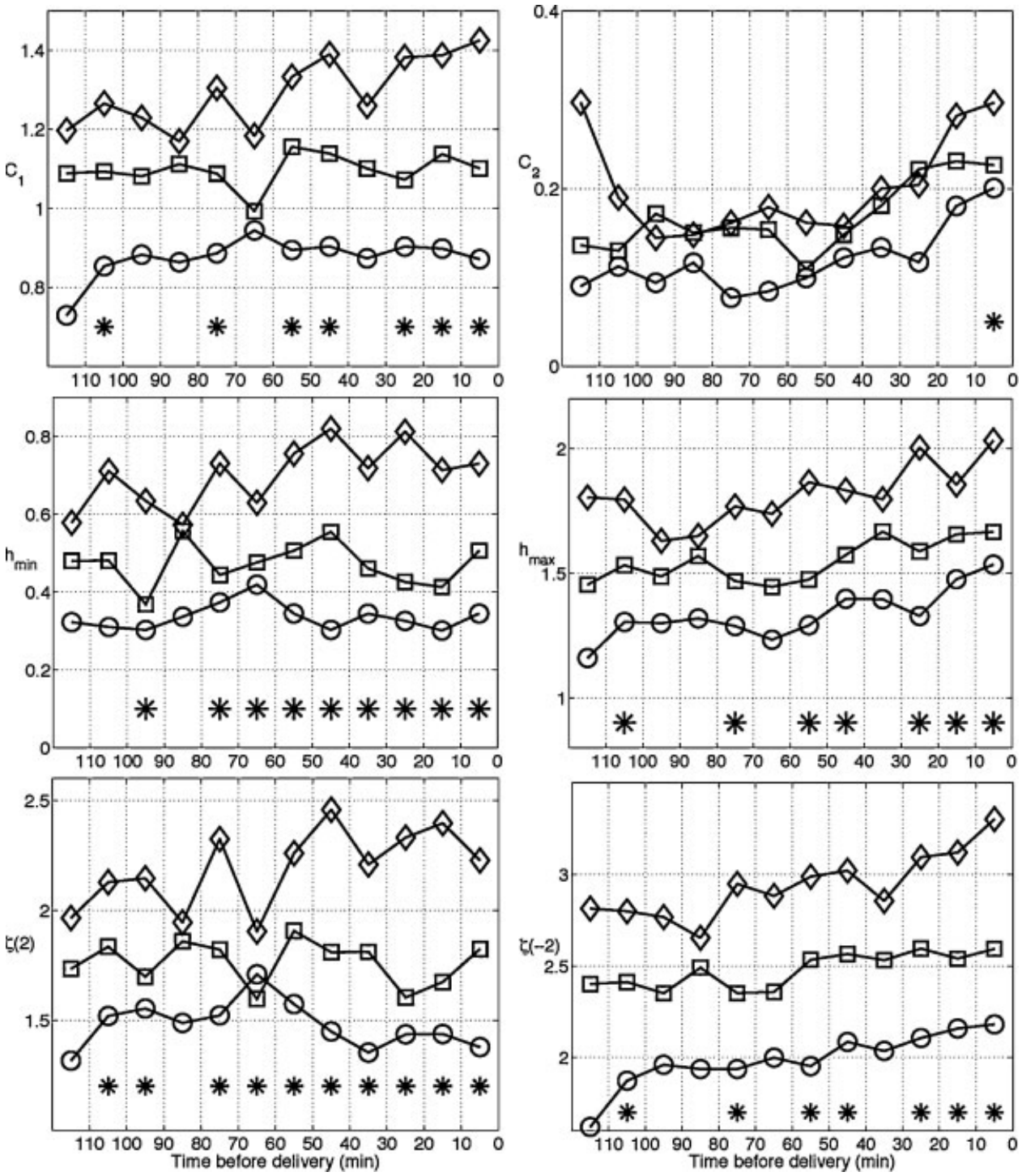

Figure 3 Time evolution of the median of the six multifractal parameters. Time evolution in group A $(\bigcirc)$, group B $(\square)$, and group $C(\diamond)$ over the last 2 hours. ${ }^{*}$ Significant difference between groups B and C.

delivery. The evolution along time of the per group medians of these six parameters is displayed in Fig. 3. For each 10-minute time window, each one of the six multifractal parameters was compared between groups B and $\mathrm{C}$ using the Wilcoxon rank sum test. Statistical significance is depicted in Fig. 3. The parameters $b_{\min }$ and zeta(2) are significantly different as early as 70 minutes before delivery. The parameters $C_{1}, b_{\mathrm{Max}}$, and zeta(-2) were significantly different 30 minutes before delivery, whereas $C_{2}$ was different only in the last 10 minutes before delivery.

\section{DISCUSSION}

This study shows that parameters stemming from multifractal analysis of fetal ECG R-R intervals are different in fetuses developing acidosis during labor and nonacidotic fetuses. All six multifractal parameters tested in this study were significantly higher in fetuses with acidosis compared with fetuses with normal umbilical cord $\mathrm{pH}$ in the last 10 minutes before delivery, regardless of FHR pattern. Interestingly, the difference was significant as early as 70 minutes before delivery using the two parameters $h_{\min }$ and zeta(2), which indicates that fetal metabolic acidosis might develop progressively or that the method can identify hypoxia preceding acidosis; $C_{2}$ was different only in the last 10 minutes, when acidosis is already present. As cases selected in this study exhibited progressive hypoxia, our results suggest that multifractal analysis should help identify both developing hypoxia and installed severe acidosis, with different parameters.

Multifractal analysis has the strength to reach results consistent with previous studies about variability using other mathematical methods, such as the classical spectral analysis, and to provide additional information 
that could help in discriminating fetuses with acidosis from nonacidotic fetuses. ${ }^{2,7,16,18}$

Consistent with previous studies and physiopathologic mechanisms, the results of this study showed that variability is lower in the acidotic fetuses when compared with the nonacidotic fetuses as demonstrated by higher values of $C_{1}, h_{\min }, h_{\mathrm{Max}}$, and zeta(2) in group C. $2,7,16,18$ Moreover, multifractal analysis focuses on characterizing the evolution of signal variability in a collection of windows of growing size, identified by the method itself according to the intrinsic data characteristics and not chosen a priori. Our results showed that windows of interest for multifractal analysis ranged from 4 to 64 seconds. This is interesting for two reasons. First, it contributes to justify and explain the limits of using short- and long-term variability to characterize variability of FHR, evaluating variability in 3.75 and 60 seconds, respectively, as described by Dawes et al. ${ }^{29}$ Multifractal analysis demonstrated that relevant information is contained not only in these two extreme bounds (4 and 64 seconds) but in a continuum of time windows in between. This may explain why short- and long-term variability can provide relevant information during pregnancy when the fetal situation is rather stable but they are not efficient to identify compromised fetuses during labor, when metabolic conditions constantly and rapidly vary. During labor, we suggest that data from all the window sizes between 4 and 60 seconds should be considered to better characterize FHR variability and therefore oxygenation status. ${ }^{11}$ Second, these windows from 4 to 64 seconds correspond to a frequency scale varying from $0.015 \mathrm{~Hz}$ to $1 \mathrm{~Hz}$, which cover the frequency domains of adult heart rate. ${ }^{8,16}$ In spectral analysis, the low-frequency domain (from 0.04 to 0.15 $\mathrm{Hz}$ ) mainly corresponds to the sympathetic activity. The high-frequency band (from 0.15 to $1 \mathrm{~Hz}$ ) corresponds mainly to the parasympathetic activity. ${ }^{8,16}$ The low- to high-frequency ratio therefore reflects the balance between the sympathetic and the parasympathetic activities. ${ }^{30-32}$ Studies from Siira et al and Salamalekis et al showed a predominant increase in the low-frequency domain and, therefore, a higher low- to high-frequency ratio in acidotic fetuses, consistent with the increase in sympathetic system activity induced by hypoxia. ${ }^{16,19,31,32}$ Interestingly, one of the multifractal parameter we studied (zeta(2)), which is related to low- to highfrequency ratio, also showed a predominant increase in sympathetic component when fetal acidosis developed. ${ }^{31-33}$

One of the strengths of multifractal analysis is also to provide multiple parameters to explore signal variability, particularly for complex signals that can definitely not be appropriately described using only one parameter. $^{22,23}$ We choose to limit the study to six parameters describing the multifractal spectrum, but the whole curve could be compared with an increased number of parameters (Fig. 1). The promise of the multifractal approach is well illustrated in the comparison between groups $\mathrm{A}$ and $\mathrm{B}$, both of which include cases with normal $\mathrm{pH}$. Unlike the regular FHR evaluation, multifractal analysis showed that these two groups were similar. The parameter $C_{1}$, which quantifies the global variability, was lower in group B than in group C. This is consistent with the reduction in variability commonly identified from visual analysis of the FHR patterns. However, the five other multifractal parameters $\left(C_{2}, h_{\min }, h_{\mathrm{Max}}\right.$, zeta(2), and zeta(-2)), which give more information about variability characteristics, were similar in both groups. These specific results highlight that the single quantitative analysis of global variability is not sufficient to predict fetal acidosis and that using other parameters that look deeper in the signal characteristics might better characterize the signal and help in acidosis diagnosis. Conversely, when comparing group B and group $\mathrm{C}$, which exhibited very different fetus acid-base status (respectively, normal $\mathrm{pH}$ and severe acidosis), the six multifractal parameters were clearly different while all FHR patterns were pathological and very similar. Pathological FHR patterns represent the most difficult situation to deal with because they can be related to severe hypoxia and usually lead to operative delivery for suspected fetal distress. Therefore, multifractal analysis could be a relevant method to identify compromised fetuses, in addition to FHR patterns, and a promising tool to assist obstetricians in decision making, helping to reduce unnecessary operative deliveries.

From a technical perspective, multifractal analysis presents the advantage of a low computational cost, allowing easy real-time analysis, essential for fetal monitoring during labor. Moreover, new technologies now allow noninvasive acquisition of the beat-to-beat $\mathrm{R}-\mathrm{R}$ interval with transabdominal ECG recording, with intact membranes and without any contraindications. Interestingly, these first results are promising even though the FHR signals were analyzed independently from uterine contractile activity, which is sometimes difficult to record because of maternal abdominal wall thickness or position during labor. And last, multifractal analysis should provide objective information, whatever the FHR patterns, limiting subjective observer interpretation. $^{34}$

\section{CONCLUSION}

This study was able to identify multifractal parameters $\left(b_{\min }\right.$ and zeta(2)) that differ in fetuses with and without developing acidosis during labor, whatever the FHR patterns, as early as 70 minutes before delivery, whereas the parameter $C_{2}$ is different only in the last 10 minutes when fetal acidosis is present. Further investigations are planned to confirm these experimental results on a large set of data. Therefore, we will investigate how multifractal 
parameters can be used, in addition to FHR, to discriminate fetuses according to the acid-base status by testing different classifier methods.

\section{ACKNOWLEDGMENT}

The Del Duca Foundation, Institut de France, and the Hospices Civils de Lyon are gratefully acknowledged for financial support. We acknowledged the help from $\mathrm{Neo}^{-}$ venta Medical with extracting the $\mathrm{R}-\mathrm{R}$ intervals from the STAN recording files.

\section{APPENDIX}

\section{Multifractal Analysis}

Multifractal analysis departs from, and hence enriches, the classical analysis of variability in two respects. First, multifractal analysis does not measure the variability in a time window of a priori chosen and fixed size, but insteadcomputes variability simultaneously over a collection of windows with different sizes. As a result, variability is not defined by the values measured within each window of different sizes but rather by the strength with which these values vary from one window size to another; strength actually measured via the so-called Hölder exponent $\mathrm{h}$, which by definition is positive (Fig. 1). When $\mathrm{h}$ is large, the variability is low and, conversely, a small value of $h$ indicates a strong variability. Second, multifractal analysis does not intend to measure the Hölder exponent $h(t)$ for each time position $t$ but instead prefers a global description of how often a specific value of $h$ is encountered in the data. This description is called the multifractal spectrum $\mathrm{D}(\mathrm{h})$. It consists of a bell-shaped curve, taking values between 0 and 1 that represent, qualitatively and heuristically, the frequency of occurrences of a given $h$ in the data. A value of $\mathrm{D}(\mathrm{h})$ close to 1 indicates that the corresponding $\mathrm{h}$ is very likely to be observed in data; conversely, values of $\mathrm{D}(\mathrm{h})$ close to 0 corresponds to rare observations of the corresponding $\mathrm{h}$. A representation of multifractal spectra computed on fetal heart rate time series is shown in Fig. 2. Moreover, the multifractal analysis used in the present contribution does not rely on the use of oscillations, but instead is based on new quantities constructed from the discrete wavelet transform coefficients of the data and referred to as the wavelet Leaders, which have been recently shown to offer solid mathematical and efficient practical frameworks permitting robust, accurate, and rich analysis of the data variability, and also to reinforce mathematical arguments underlying the multifractal formalism. ${ }^{14,15,26}$

Practically to obtain $\mathrm{D}(\mathrm{h})$ spectrum from data, intermediate quantities zeta(q), termed as the scaling exponents, are necessary. Indeed, in essence, multifractal analysis relies on the fact that the time average (denoted as $\langle>$ ) of the q-th power of the wavelet Leaders computed from analyzing window of any arbitrary size "a" behave as a power law with respect to "a": $\left.<\mathrm{L} \_\mathrm{X}(\mathrm{a})^{\mathrm{q}}\right\rangle, \approx \mathrm{a}^{\text {zeta(q) }}$. The scaling exponents zeta (q) can hence be measured from a linear regression in a log $\log$ diagram, performed over a range of window size "a" where the power law behavior holds. This is illustrated in Fig. 3. Then, $\mathrm{D}(\mathrm{h})$ is obtained by applying a Legendre transform to the function zeta(q). Multifractal analysis hence characterizes the variability of data via both the bell-shaped curve $\mathrm{D}(\mathrm{h})$ or the scaling exponent function zeta(q). To compare multifractal spectra $\mathrm{D}(\mathrm{h})$ or scaling exponents zeta(q) of different signals, a limited number of multifractal parameters have been selected. We retained six of them, referred to as $\mathrm{C} 1, \mathrm{C} 2, \mathrm{~h}_{\min }, \mathrm{h}_{\mathrm{Max}}$, zeta(2), and zeta(-2), illustrated in Fig. 1 and defined as follows. The parameter $\mathrm{C} 1$ corresponds to the value of $\mathrm{h}$ where $\mathrm{D}(\mathrm{h})$ is maximum. It can be read as the "almost sure" variability of the analyzed data. A decrease in $\mathrm{C} 1$ indicates an increase in variability. The parameter $\mathrm{C} 2$ corresponds to the width of $\mathrm{D}(\mathrm{h})$ around its maximum and indicates how widely the variability that can be measured in the data, can depart from the typical value $\mathrm{C} 1$. The larger $\mathrm{C} 2$ is, the larger the deviation from $\mathrm{C} 1$ will be. In other words, when $\mathrm{C} 2$ is small, the variability of the data is of the same order along time, whereas a high $\mathrm{C} 2$ indicates that portions of the signal are much more variable and that others are much less variable, compared to the typical $\mathrm{C} 1$ value. The parameters $\mathrm{h}_{\min }$ and $h_{\max }$ correspond respectively to the largest and smallest variabilities that can be observed in the data. We choose to retain the parameter zeta (2) as it can be theoretically related to Fourier analysis and spectrum estimation. The larger that zeta (2) is, the weaker the variability in the data. Multifractal theory also states that the function zeta(q) needs to be computed for both positive and negative values of $\mathrm{q}$. Therefore, we included zeta $(-2)$ as a sixth mutlifractal parameter.

\section{REFERENCES}

1. Nelson KB, Dambrosia JM, Ting TY, Grether JK. Uncertain value of electronic fetal monitoring in predicting cerebral palsy. N Engl J Med 1996;334:613-618

2. Low JA, Victory R, Derrick EJ. Predictive value of electronic fetal monitoring for intrapartum fetal asphyxia with metabolic acidosis. Obstet Gynecol 1999;93:285-291

3. East CE, Leader LR, Sheehan P, Henshall NE, Colditz PB. Intrapartum fetal scalp lactate sampling for fetal assessment in the presence of a non-reassuring fetal heart rate trace. Cochrane Database Syst Rev 2010;3:CD006174

4. Amer-Wåhlin I, Hellsten C, Norén H, et al. Cardiotocography only versus cardiotocography plus ST analysis of fetal electrocardiogram for intrapartum fetal monitoring: a Swedish randomised controlled trial. Lancet 2001;358: 534-538

5. Ojala K, Vääräsmäki $M$, Mäkikallio K, Valkama M, Tekay A. A comparison of intrapartum automated fetal 
electrocardiography and conventional cardiotocography-a randomised controlled study. BJOG 2006;113:419-423

6. Vayssière C, David E, Meyer N, et al. A French randomized controlled trial of ST-segment analysis in a population with abnormal cardiotocograms during labor. Am J Obstet Gynecol 2007;197:299; e1-e6

7. Williams KP, Galerneau F. Intrapartum fetal heart rate patterns in the prediction of neonatal acidemia. Am J Obstet Gynecol 2003;188:820-823

8. Akselrod S, Gordon D, Ubel FA, Shannon DC, Berger AC, Cohen RJ. Power spectrum analysis of heart rate fluctuation: a quantitative probe of beat-to-beat cardiovascular control. Science 1981;213:220-222

9. Humeau A, Chapeau-Blondeau F, Rousseau D, Rousseau P, Trzepizur W, Abraham P. Multifractality, sample entropy, and wavelet analyses for age-related changes in the peripheral cardiovascular system: preliminary results. Med Phys 2008;35: 717-723

10. Lipsitz LA, Mietus J, Moody GB, Goldberger AL. Spectral characteristics of heart rate variability before and during postural tilt. Relations to aging and risk of syncope. Circulation 1990;81:1803-1810

11. Goldberger AL, Amaral LA, Hausdorff JM, Ivanov PCh, Peng CK, Stanley HE. Fractal dynamics in physiology: alterations with disease and aging. Proc Natl Acad Sci U S A 2002;99(Suppl 1):2466-2472

12. Ivanov PC, Amaral LA, Goldberger AL, et al. Multifractality in human heartbeat dynamics. Nature 1999;399:461-465

13. Dawes GS, Rosevear SK, Pello LC, Moulden M, Redman CW. Computerized analysis of episodic changes in fetal heart rate variation in early labor. Am J Obstet Gynecol 1991;165: 618-624

14. Riedi RH. Multifractal processes. In: Dourkhan P, Oppenheim G, Taqqu NS, eds. Theory and Applications of Long Range Dependence. Basel, Switzerland: Birkhauser; 2003: 625-716

15. Jaffard S. Wavelet techniques in multifractal analysis in fractal geometry and applications: a jubilee of Benoit Mandelbrot. In: Lapidus $M$ van Frankenhuijsen M, ed. Proceedings of Symposia in Pure Mathematics. Providence, RI: ANS Bookstore; 2004:91-152

16. Siira SM, Ojala TH, Vahlberg TJ, et al. Marked fetal acidosis and specific changes in power spectrum analysis of fetal heart rate variability recorded during the last hour of labour. BJOG 2005;112:418-423

17. Chung DY, Sim YB, Park KT, Yi SH, Shin JC, Kim SP. Spectral analysis of fetal heart rate variability as a predictor of intrapartum fetal distress. Int J Gynaecol Obstet 2001;73:109116

18. Rantonen T, Ekholm E, Siira S, et al. Periodic spectral components of fetal heart rate variability reflect the changes in cord arterial base deficit values: a preliminary report. Early Hum Dev 2001;60:233-238

19. Salamalekis E, Hintipas E, Salloum I, et al. Computerized analysis of fetal heart rate variability using the matching pursuit technique as an indicator of fetal hypoxia during labor. J Matern Fetal Neonatal Med 2006;19:165-169

20. Weiner Z, Farmakides G, Schulman H, Kellner L, Plancher S, Maulik D. Computerized analysis of fetal heart rate variation in postterm pregnancy: prediction of intrapartum fetal distress and fetal acidosis. Am J Obstet Gynecol 1994;171:1132-1138

21. Hopkins P, Outram N, Löfgren N, Ifeachor EC, Rosén KG. A comparative study of fetal rate variability analysis techniques. Conf Proc IEEE Eng Ned Biol Soc 2006;1: 1784-1787

22. Lopes R, Betrouni N. Fractal and multifractal analysis: a review. Med Image Anal 2009;13:634-649

23. Nakamura T, Horio H, Chiba Y. Local holder exponent analysis of heart rate variability in preterm infants. IEEE Trans Biomed Eng 2006;53:83-88

24. Hanson MA. The importance of baro- and chemoreflexes in the control of the fetal cardiovascular system. J Dev Physiol 1988;10:491-511

25. Macones GA, Hankins GD, Spong CY, Hauth J, Moore T. The 2008 National Institute of Child Health and Human Development workshop report on electronic fetal monitoring: update on definitions, interpretation, and research guidelines. Obstet Gynecol 2008;112:661-666

26. Abry P, Wendt H, Jaffard S, et al. Methodology for multifractal analysis of heart rate variability: from $\mathrm{LF} / \mathrm{HF}$ ratio to wavelet leaders. Proc of Engineering in Medicine and Biology Conference (IEEE-ENBC), Buenos Aires, Argentina; 2010:106-109

27. Wendt H, Abry P, Jaffard S. Bootstrap for empirical multifractal analysis. IEEE Signal Process Mag 2007;24:38-48

28. Abry P, Goncalves P, Flandrin P. Wavelets, spectrum analysis and $1 / \mathrm{f}$-processes. Wavelets and statistics. In: Antoniadis A Oppenheim G, ed. Lecture Notes in Statistics. New York: Springer-Verlag; 1995:15-30

29. Dawes GS, Redman CWG, Smith JH. Improvements in the registration and analysis of fetal heart rate records at the bedside. Br J Obstet Gynaecol 1985;92:317-325

30. Randall DC, Brown DR, Raisch RM, Yingling JD, Randall WC. SA nodal parasympathectomy delineates autonomic control of heart rate power spectrum. Am J Physiol 1991; 260(3 Pt 2):H985-H988

31. Alonso-Spilsbury M, Mota-Rojas D, Villanueva-García D, et al. Perinatal asphyxia pathophysiology in pig and human: a review. Anim Reprod Sci 2005;90(1-2):1-30

32. Jensen A, Garnier Y, Berger R. Dynamics of fetal circulatory responses to hypoxia and asphyxia. Eur J Obstet Gynecol Reprod Biol 1999;84:155-172

33. Yum MK, Kim JH. A very-short-term intermittency of fetal heart rates and developmental milestone. Pediatr Res 2003;53: 915-919

34. Vayssière C, Tsatsaris V, Pirrello O, Cristini C, Arnaud C, Goffinet F. Inter-observer agreement in clinical decisionmaking for abnormal cardiotocogram (CTG) during labour: a comparison between CTG and CTG plus STAN. BJOG 2009;116:1081-1087; discussion 1087-1088 